\title{
Stability and Reproducibility of Radiometric Properties of Light Curing Units (LCUs). Part II: LED LCUs
}

\author{
María del Mar PÉREZ ${ }^{1}$, Francisco PÉREZ-OCÓN, Cristina LUCENA-MARTÍN² and Rosa PULGAR ${ }^{2}$ \\ ${ }^{1}$ Department of Optics, Science Faculty, University of Granada, Campus Fuentenueva s/n, 18071, Granada, Spain \\ ${ }^{2}$ Department of Stomatology, Faculty of Dentistry, University of Granada, Campus Cartuja s/n, 18071, Granada, Spain \\ Corresponding author, M. M. PÉREZ; E-mail: mmperez@ugr.es
}

Received June 17, 2007/Accepted October 20, 2007

\begin{abstract}
The present work is the second part of a study on the stability and reproducibility of the energy and spectral emission of some light curing units (LCUs). In this part, Part II, LED LCUs were investigated. Results revealed that these devices presented high stability and reproducibility in terms of their spectral emission, with values of VAF (variance accounting for) coefficient calculated from the Cauchy-Schwarz inequality all close to $100 \%$. With respect to energy stability, the LED LCUs presented energy stability except for the third-generation LED LCUs which have several LEDs. For these devices, the law of reciprocity was not fulfilled as irradiance was not constant over exposure time. This result should be taken into account both in works examining the polymerization kinetics of dental materials as well as when these LED LCUs are used in clinical practice.
\end{abstract}

Keywords: LED light curing units (LED LCUs), Radiometric properties, Spectral emission

\section{INTRODUCTION}

Development in light-emitting diodes (LEDs) in recent years has generated a significant growth market with completely new applications. Amongst which, blue LEDs have been proposed for curing light-activated dental materials ${ }^{1,2}$. The spectral output of certain blue LEDs falls within the absorption spectrum of camphorquinone (CQ). This technology hence solves some problems inherent in dental light curing units (LCUs) based on halogen technology to polymerize oral biomaterials. The advantages ${ }^{3)}$ include: (1) narrow spectrum eliminates the light filter system required by other technologies; (2) efficiently converts electricity into light with less heat generation, and subsequently less cooling is required; (3) long service life as it routinely functions on battery-charging cycles and battery life; and (4) little or no degradation over time as compared to the degradation of lamp, filter, and reflector with time in conventional halogen units. Further, it has been recently demonstrated that blue GaN LED has the potential to cure composite resins to the effect of exceeding the minimum requirements of ISO standards for depth of cure and tensile strength ${ }^{4}$. In view of the favourable results obtained, its performance can be considered clinically satisfactory ${ }^{5-7)}$.

Nevertheless, its narrow emission width can be considered more as a disadvantage than an advantage. In fact, LED technology for dental curing uses a wavelength spectrum of $450 \mathrm{~nm}$ to $500 \mathrm{~nm}$, which makes LED technology useless for any photoinitiator with absorption peaks below $450 \mathrm{~nm}$ - such as phenyl-propanedione, PPD, which has an absorption peak of $398 \mathrm{~nm}$, Lucirin at $381 \mathrm{~nm}$, or Irgacure with maximum peak at $370 \mathrm{~nm}^{8}$.
Since its introduction into the market, LED LCUs have evolved considerably, especially in terms of irradiance. The so-called first-generation LED LCUs presented an irradiance of $400 \mathrm{~mW} / \mathrm{cm}^{2}$ and a power output of $1 \mathrm{~W}$. The so-called second generation reached $800 \mathrm{~mW} / \mathrm{cm}^{2}$ and $5 \mathrm{~W}$, and the third generation, which emerged recently, exceeds 1.100 $\mathrm{mW} / \mathrm{cm}^{2}$ and $8 \mathrm{~W}$. To date, study of dental LCUs has been approached from the analysis of their effectiveness to polymerize a certain dental material more than from the standpoint of evaluating their radiometric characteristics largely responsible for this effectiveness ${ }^{9-12}$. Moreover, very frequently, these studies that compared LCUs or light curing protocols tended to focus on the different radiant exposure conditions or the final properties of the different materials, hence hampering the analysis and comparison of the results ${ }^{13)}$. Consequently, it has been stated that LED LCUs polymerize more deeply than halogen lamps because their spectrum coincides with that of $\mathrm{CQ}$, or that they are more efficient on an equalenergy basis ${ }^{14}$.

It is noteworthy that the polymerization of a composite depends not only on the amount of energy supplied, but also on the material's formulation ${ }^{15}$. In other words, results of these comparative studies cannot be indiscriminately and misleadingly extrapolated from one material to another. For this reason, if a study seeks to evaluate an LCU and compare one type of LCU versus another, the study needs to take into account the characteristics of the device itself and, specifically, its radiometric and spectral characteristics ${ }^{9,16)}$.

The aim of the present study, therefore, was to evaluate the stability and reproducibility of the radiometric properties of present LCUs, namely QHT 
and LED LCUs. This work has been divided into two parts. In Part I, four QTH LCUs were investigated. In Part II, which was the present study, the focus was on LED LCUs. The first working hypothesis tested was that LED LCUs present spectral stability - that is, the shape of the emission curve as a function of exposure time does not vary. The second hypothesis tested was that LED LCUs present energy stability - that is, their total irradiance remains constant over exposure time.

\section{MATERIALS AND METHODS}

\section{Characterization of the LCUs}

In this study, seven commercial LED LCUs, provided by the manufacturers, were investigated. They were Bluephase (SN 1523676, Ivoclar Vivadent, Schaan, Liechtenstein), SmartLite PS and SmartLite IQ (SN AA00147 and 8878 respectively, Dentsply, Konstanz, Germany), MiniLED (SN 196-10470, cordless LED curing light, Acteon Group, Bordeaux, France), L.E. Demetron (SN 70048224, Demetron, Division of Kerr Corp., Danbury, CT, USA), UltraLume2 LED and UltraLume 5 LED (SN 004078 and 000114 respectively, Ultradent Products, South Jordan, UT, USA). Table 1 shows the technical characteristics specified by the manufacturers. Based on the irradiance infor- mation given by each LED LCU's manufacturer and the respective output face dimensions, light emission flux was thereby calculated.

The emission spectrum of the light source, the spectral irradiance for each wavelength, and the total irradiance were determined using the same instrument as in Part I. The experimental conditions were the same as those in Part I, and likewise a total radiant exposure of $18 \mathrm{~J} / \mathrm{cm}^{2}$ was used.

\section{Stability and reproducibility evaluation}

To evaluate the stability and reproducibility of the spectral emissions of LED LCUs, correlation coefficients using Cauchy - Schwarz's inequality and VAF (variance accounting for) values were calculated. Intra-protocol and inter-protocol VAF values were then obtained as described in Part I.

To complete our stability and reproducibility analysis of the radiometric properties of each LCU, we also had to evaluate whether the total irradiance remained constant over exposure time according to this protocol. This study was conducted with three successive replicates of the same protocol. Based on the experimental data, a straight-line fit and regression coefficient for the three replicates of each light curing protocol were acquired and examined.

Table 1 Measured technical details of LCUs used in this study

\begin{tabular}{|c|c|c|c|c|c|c|c|}
\hline $\mathrm{LCU}$ & Blue Phase & L.E.Demetron & MiniLED & Smart Lite I.Q. & Smart Lite PS & UltraLume 2LED & UltraLume 5LED \\
\hline Light source & 1 LED & 4 LEDs & 1 LED & 1 LED & 1 LED & 2 LEDs & 5 LEDs \\
\hline $\begin{array}{l}\text { Experimental peak } \\
\text { emission wavelength } \\
(\mathrm{nm})\end{array}$ & 452 & 452 & 452 & 456 & 456 & 448 & $\begin{array}{l}456 \\
400\end{array}$ \\
\hline $\begin{array}{l}\text { Manufacturer light } \\
\text { emission flux (mW) }\end{array}$ & 553 & 293 & 552 & 511 & 455 & 561 & 918 \\
\hline $\begin{array}{l}\text { Experimental light } \\
\text { emission flux (mW) }\end{array}$ & 555 & 379 & 669 & 995 & 508 & 1594 & 993 \\
\hline $\begin{array}{l}\text { Output-face light guide } \\
\text { tip or window (mm) }\end{array}$ & 8 & 6.43 & 7.5 & 8.5 & 8 & 6.43 & 6.43 \\
\hline $\begin{array}{l}\text { Irradiance (manufac- } \\
\text { turer) }\left(\mathrm{mW} / \mathrm{cm}^{2}\right)\end{array}$ & 1100 & 800 & 1250 & 900 & 950 & $>550$ & $>900$ \\
\hline $\begin{array}{l}\text { Irradiance (experimen- } \\
\text { tal) }\left(\mathrm{mW} / \mathrm{cm}^{2}\right)\end{array}$ & 1104 & 1440 & 1514 & 1753 & 1010 & 1561 & 973 \\
\hline Irradiance time (s) & 16 & 23 & 14 & $20 \mathrm{~s}$ & 19 & 33 & 20 \\
\hline $\begin{array}{l}\text { Total radiant exposure } \\
\text { (manufacturer) }\left(\mathrm{J} / \mathrm{cm}^{2}\right)\end{array}$ & 17.6 & 18.4 & 17.5 & 18 & 18 & 18.2 & 18 \\
\hline $\begin{array}{l}\text { Total radiant exposure } \\
\text { (experimental) }\left(\mathrm{J} / \mathrm{cm}^{2}\right)\end{array}$ & 17.7 & 26.8 & 21.2 & 35.1 & 19.2 & 51.5 & 19.5 \\
\hline
\end{tabular}




\section{RESULTS}

Table 1 shows the experimental values of the spectral and radiometric characteristics of each LED LCU examined in the present study: peak emission, light emission flux, total irradiance (mean value of three measurements), and total radiant exposure. As in Part I, the uncertainty that accompanied all the experimental measurements of irradiance was $6 \%$.

Figure 1 shows the spectral irradiances of the seven LED LCUs used in this study. The thick solid line denotes the absorption spectrum of CQ (in arbitrary units). It is worth noting that, as reflected in Fig. 1, UltraLume5 LED exhibited two emission peaks at 400 and $456 \mathrm{~nm}$, given that it had two different types of LEDs.

Table 2 shows the maximum and minimum intra-protocol and inter-protocol VAF values for each of the LCUs. Maximum intra-protocol VAF value was 99.99\%. The most unfavourable intra-protocol VAF value was $97.37 \%$, obtained for the first replicate of the protocol corresponding to UltraLume5

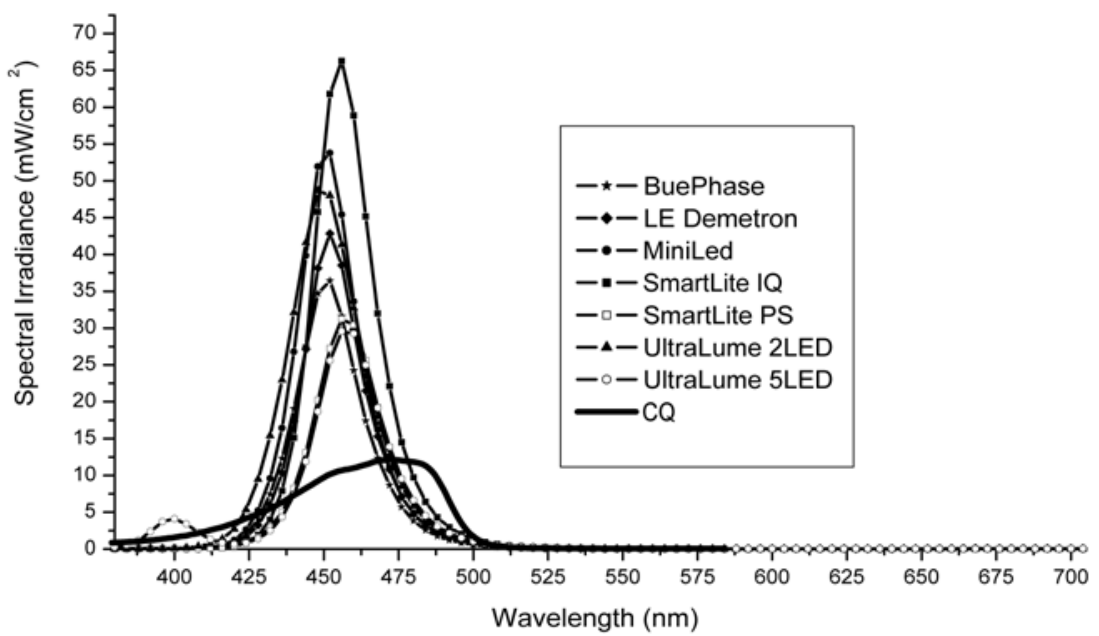

Fig. 1 Spectral irradiances of the LED LCUs and the absorption spectrum of camphorquinone (in arbitrary unit).

Table 2 Maximum and minimum intra-protocol and inter-protocol VAF values of each LCU

\begin{tabular}{|c|c|c|c|}
\hline $\mathrm{LCU}$ & Replicates & VAF intra-protocol & VAF inter-protocol \\
\hline & & Maximum-Minimum & Maximum-Minimum \\
\hline \multirow{3}{*}{ Blue Phase } & 1. & $99.99 \%-99.85 \%$ & \multirow{3}{*}{$99.99 \%-99.95 \%$} \\
\hline & 2 & $99.99 \%-99.84 \%$ & \\
\hline & 3 & $99.99 \%-99.82 \%$ & \\
\hline \multirow{3}{*}{ L.E. Demetron } & 1. & $99.99 \%-99.42 \%$ & \multirow{3}{*}{$99.94 \%-99.35 \%$} \\
\hline & 2 & $99.99 \%-99.04 \%$ & \\
\hline & 3 & $99.99 \%-99.54 \%$ & \\
\hline \multirow{3}{*}{ MiniLed } & 1. & $99.99 \%-99.76 \%$ & \multirow{3}{*}{$99.89 \%-99.56 \%$} \\
\hline & 2 & $99.99 \%-99.80 \%$ & \\
\hline & 3 & $99.99 \%-99.81 \%$ & \\
\hline \multirow{3}{*}{ SmartLite I. Q. } & 1. & $99.99 \%-99.94 \%$ & \multirow{3}{*}{$99.99 \%-99.96 \%$} \\
\hline & 2 & $99.99 \%-99.93 \%$ & \\
\hline & 3 & $99.99 \%-99.90 \%$ & \\
\hline \multirow{3}{*}{ SmartLite S. P. } & 1. & $99.99 \%-99.80 \%$ & \multirow{3}{*}{$99.98 \%-99.70 \%$} \\
\hline & 2 & $99.99 \%-99.80 \%$ & \\
\hline & 3 & $99.99 \%-99.84 \%$ & \\
\hline \multirow{3}{*}{$\begin{array}{l}\text { UltraLume } \\
\text { 2LED }\end{array}$} & 1. & $99.99 \%-99.73 \%$ & \multirow{3}{*}{$99.99 \%-99.82 \%$} \\
\hline & 2 & $99.99 \%-99.97 \%$ & \\
\hline & 3 & $99.99 \%-99.73 \%$ & \\
\hline \multirow{3}{*}{$\begin{array}{l}\text { UltraLume } \\
\text { 5LED }\end{array}$} & 1. & $99.99 \%-97.37 \%$ & \multirow{3}{*}{$99.96 \%-97.73 \%$} \\
\hline & 2 & $99.99 \%-99.65 \%$ & \\
\hline & 3 & $99.99 \%-99.93 \%$ & \\
\hline
\end{tabular}


LED. It warrants highlighting that $97.73 \%$ of UltraLume5 LED was an exceptional case, as the rest of the minimum values exceeded $99.00 \%$.

With regard to inter-protocol VAF values, it should also be emphasised that the maximum VAF value was $99.99 \%$, while the minimum was $97.73 \%$

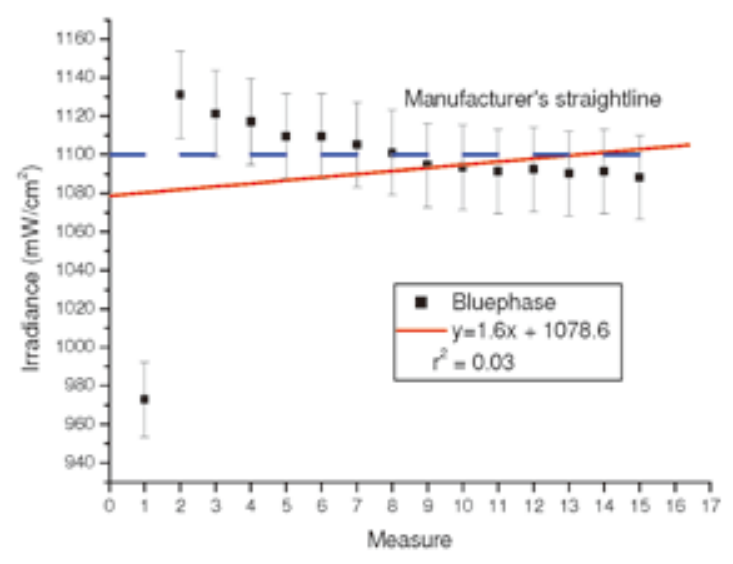

(2a)

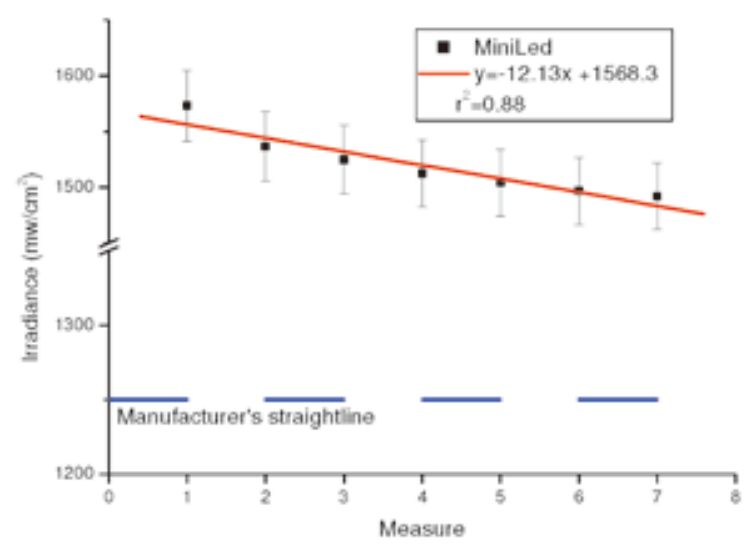

(2b)

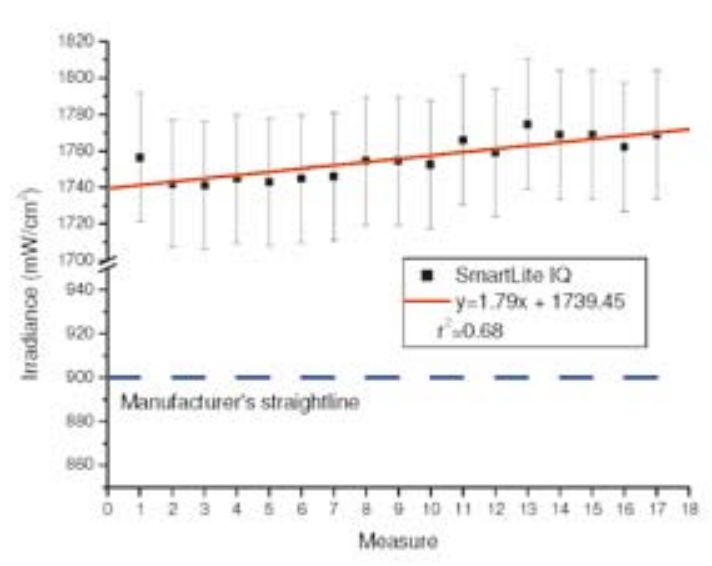

(2c) in the case of UltraLume5 LED. As with the intraprotocol VAF value, the latter result was an exceptional case as all the other minimum values exceeded $99.00 \%$.

Figures $2 \mathrm{a}-\mathrm{e}$ show the total irradiance values for Bluephase, MiniLED, SmartLite PS, SmartLite

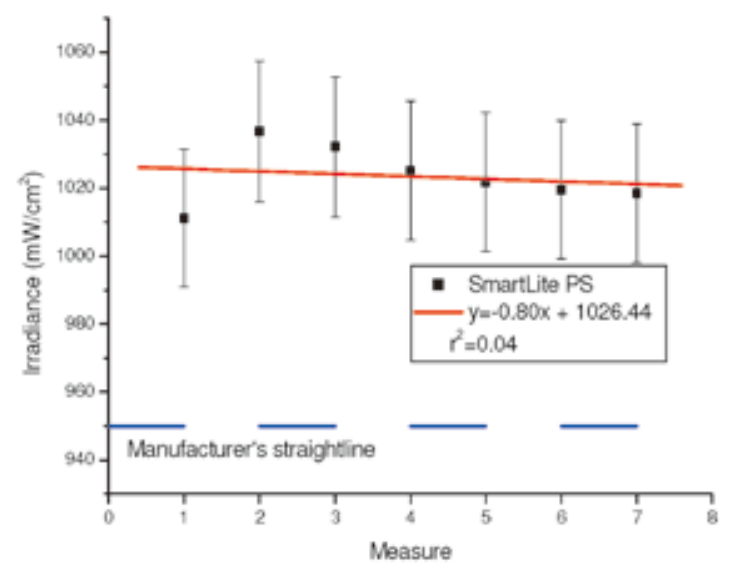

(2d)

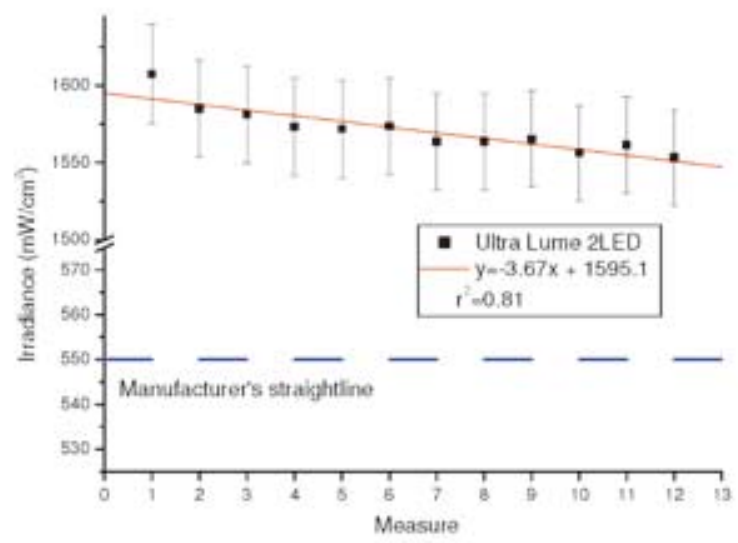

(2e)

Fig. 2 Total irradiance values of Bluephase (a), MiniLED (b), SmartLite IQ (c), SmartLite PS (d), and UltraLume2 LED (e), as well as straight lines drawn from manufacturers' and experimental values. The intervals represent instrumental errors. 


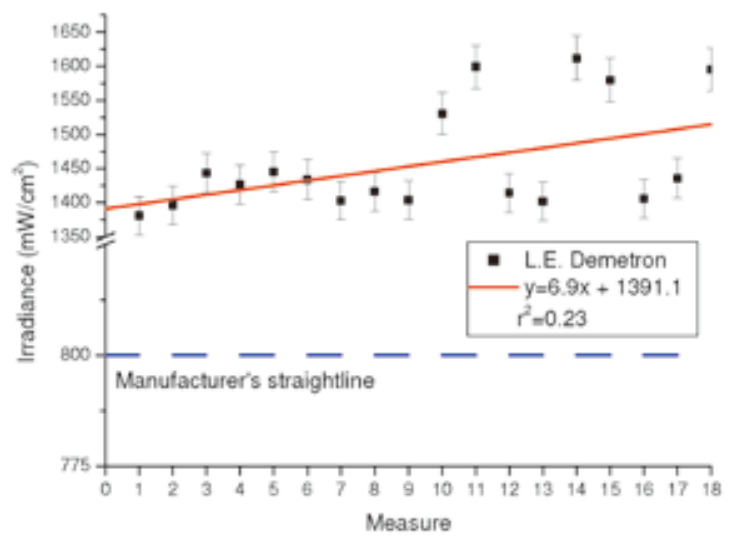

(3a)

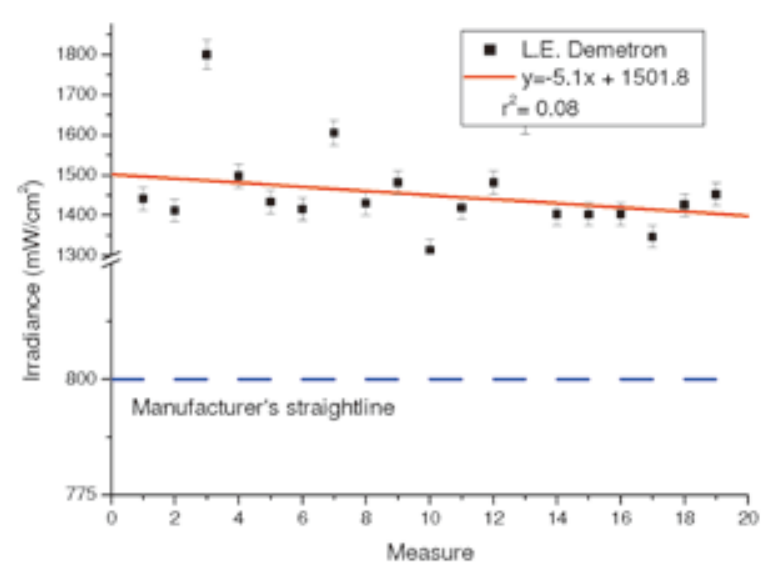

(3b)

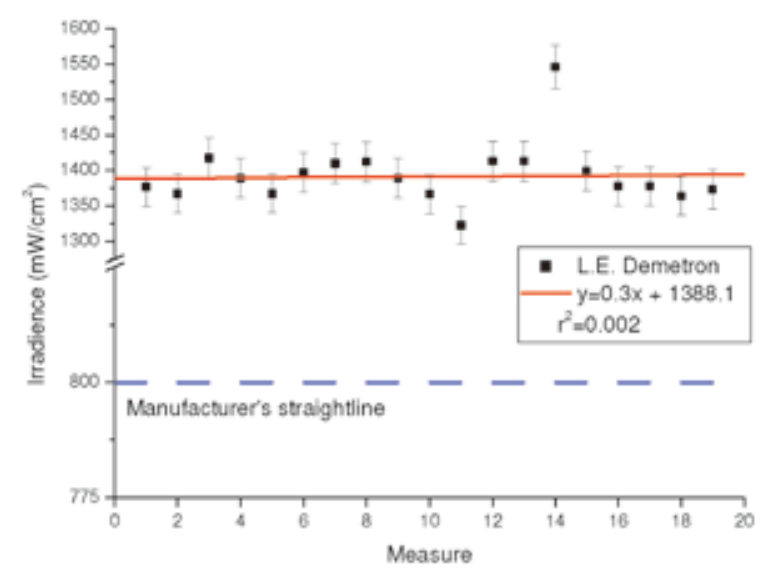

(3c)

Fig. 3 Total irradiance values of L.E. Demetron as well as straight lines drawn from manufacturer's values and experimental values for the three replicates. The intervals represent instrumental errors.

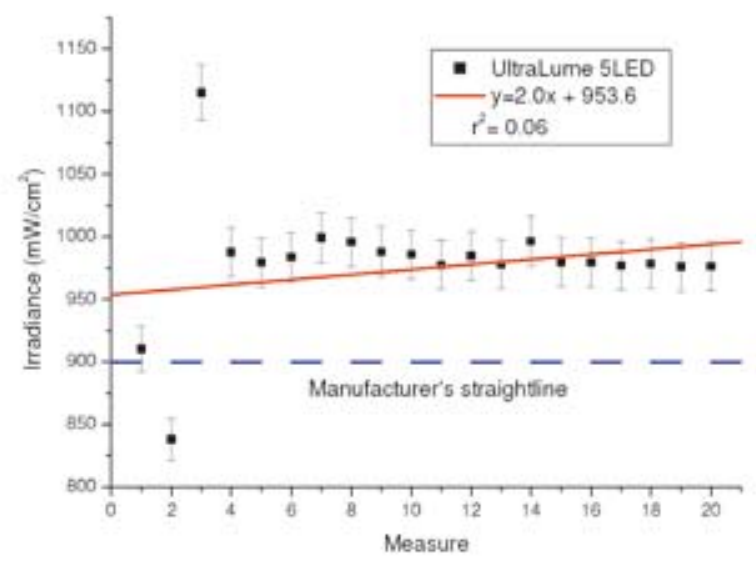

(4a)

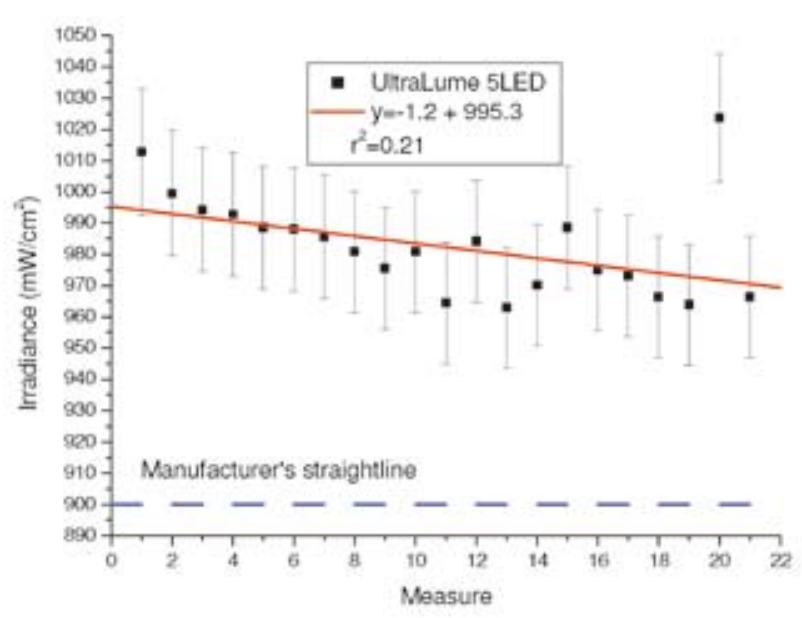

(4b)

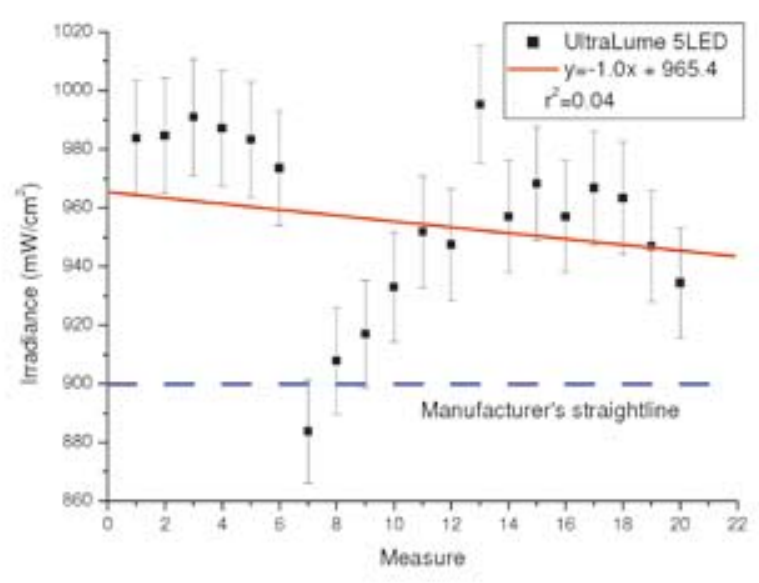

(4c)

Fig. 4 Total irradiance values of UltraLume5 LED as well as straight lines drawn from manufacturer's values and experimental values for the three replicates. The intervals represent instrumental errors. 
IQ, and UltraLume2 LED LCUs, whereby temporal stability could be evaluated. Each LCU was represented by only one of the three replicates, given the similarity of the three results for each of these five LCUs. In these figures were also shown the equations of the theoretical straight lines and the manufacturers' straight lines.

Figures $3 \mathrm{a}-\mathrm{c}$ and $4 \mathrm{a}-\mathrm{c}$ show the results of the three replicates performed for L.E. Demetron and UltraLume5 LED LCUs respectively. With these two devices, the three replicates performed are shown because of the divergence among them. As in Figs. 2a-e, equations of the corresponding theoretical straight lines for each of the three measurements and the manufacturers' straight lines are also shown.

\section{DISCUSSION}

In the present study, all the LCUs examined yielded a maximum intra-protocol VAF value of $99.99 \%$, with minimum values close to the maximum and statistically very high, indicating that the LCUs, in terms of spectral emission, presented high stability like QTH LCUs.

With respect to inter-protocol VAF values (fourth column of Table 2), conclusions similar to those for intra-protocol could be drawn. It was only in the case of UltraLume5 LED LCU, where one replicate registered a spectral emission curve that differed slightly from the other two replicates. Despite being so, the differences were not great and thus the corresponding inter-protocol VAF value was 97.73\%. As indicated in Part I of the work with QTH LCUs, the inter-protocol values obtained herein indicated the high reproducibility of the LED LCUs with regard to their spectral emission.

In light of the VAF results obtained, it could be affirmed that the LED LCUs studied exhibited stability and reproducibility in terms of spectral emission. On this ground, the first hypothesis of our research was verified and accepted.

With LED LCUs, spectral stability is even more important than with QTH LCUs. Owing to a narrower spectral width, a small variation in emission could translate into a withdrawal from the absorption peak of the photoinitiator. However, with QTH LCUs, the spectral width is great and the problem is not as critical. In summary, we can assess whether the current light curing units guarantee that during the time corresponding to a clinical protocol or to protocols close together in time, the spectral range and peak remain constant.

With respect to energy stability, concordance was found only with Bluephase (difference $<6 \%$ ) after comparing the experimental irradiance values with the theoretical ones provided by the manufacturers.
For SmartLite PS, the difference was 6\%. For most of the LCUs, this difference was far greater - which also occurred in Part I for QTH LCUs. On this note, the results of SmartLite IQ and UltraLume2 LED should in particular be highlighted as their experimental irradiance values exceeded the manufacturers' values by $190 \%$ and $280 \%$ respectively. These differences were of the order of magnitude of those found for QTH LCUs (175\% and 230\% for Bluelight and Spectrum 800 respectively).

Exposure time of each LCU was calculated from the irradiance value given by the respective manufacturer. Consequently, the quantity of energy that the device provided experimentally was far higher than the amount presumed to be applied following an established protocol, as reflected in the last two rows of Table $1\left(18 \mathrm{~J} / \mathrm{cm}^{2}\right.$ against $35.1 \mathrm{~J} / \mathrm{cm}^{2}$ for SmartLite IQ or $18.2 \mathrm{~J} / \mathrm{cm}^{2}$ against $51.5 \mathrm{~J} / \mathrm{cm}^{2}$ for UltraLume2 LED). These high amounts of energy bore practical implications for the polymerization of dental composites and the biological effects of visible light.

First, it has been unequivocally demonstrated that both irradiance and radiant exposure significantly impact the polymerization kinetics of lightcured resin composites ${ }^{17)}$, as well as the mechanical properties and cure characteristics of the resulting polymer ${ }^{18)}$. Next, there is also a prevailing concern about the biological effects of short-wavelength light (within the visible spectrum). To date, it has been affirmed that it is not harmless - whereby effects at the cellular level are dose- and wavelengthdependent $^{19)}$. Nonetheless, given the meagre information currently available on this subject, future research should be undertaken to clarify these biological effects.

Concerning the discrepancies observed between the experimental irradiance values and those given by the manufacturers, it has been reported in Part I that these discrepancies might be attributed to the different radiometric devices reporting different irradiance values, especially with dental radiometers ${ }^{20,21}$. On this note, our results for LED LCUs from the present study further supported a recent recommendation $^{9)}$ on the need for accurate measurement and precise reporting of radiometric quantities employed in the photoactivation process.

Figures $2 \mathrm{a}-\mathrm{e}$ show the results for LED LCUs which had very similar values for the three replicates of the same protocol: Bluephase, MiniLED, SmartLite PS, SmartLite IQ, and UltraLume2 LED. As in Part I, the regression coefficient values indicated that the experimental values did not closely fit a straight line, although they differed from one LCU to another. Furthermore, some devices presented an initial value below the mean (Bluephase and SmartLite PS), while others presented an initial value above the mean 
(MiniLED and UltraLume2 LED).

With MiniLED, SmartLite PS, SmartLite IQ, and UltraLume2 LED LCUs, instrumental error could be taken into account such that a straight line could be drawn with a zero slope and ordinate at the origin within the uncertainty values of the means of these three devices. Thus, these four LCUs could be considered energetically stable over time after taking into account their associated error. However, even if they were deemed energetically stable as indicated above, the negative slope showed a fall in irradiance value over time. Electronically, the decline was due to heating up of proton-neutron pairing by high-intensity LEDs and which is generally enclosed in a very small capsule with a refrigeration system consisting of a small ventilator. Nevertheless, this slight descent was not found for SmartLite IQ LCU. With this device, the values fluctuated without a definite trend.

If the first measurement were to be eliminated, as was done in Part I for some halogen LCUs, the straight line (i.e., the regression line) for Bluephase, for instance, was $\mathrm{y}=-3.01(1.40) \mathrm{x}+1128.22(13.90)$ and $p<0.0001$. As shown in Fig. 5, when the initial value was disregarded considering its associated error, the device showed good energy stability over time. Nevertheless, with LED LCUs, it was concluded that the initial irradiance value is critical in LCU stability, as was the case with QTH LCUs in Part I of the work.

For L.E. Demetron (Figs. 3a-c) and UltraLume5 LED (Figs. $4 \mathrm{a}-\mathrm{c}$ ) LCUs, they did not show a similar irradiance pattern over time for the three replicates. Based on the results for Parts I and II of our work, QTH LCUs as well as LED LCUs showed increasing and decreasing irradiance trends over time, and where stability could be considered acceptable if the first irradiance value were eliminated. However, for these two LCUs, their irradiance values over exposure time varied in an apparently random way. Furthermore, for each of the three replicates pertaining to the same protocol, there was no best-fit straight line. This occurred despite taking into account the instrumental errors or eliminating the initial irradiance value, hence showing the absence of energy stability for these two LCUs.

It should be highlighted that these two LCUs had four and five LEDs against the rest of the LED LCUs, which had only one LED and two for UltraLume2 LED. Indeed, poor energy stability is associated with LCUs that have more than one LED, since they are encapsulated or integrated. In view of these unfavourable energy stability results, the second hypothesis of the present research was rejected. In other words, the most modern LCUs presented the worst results in terms of energy stability of all the devices studied, including QTH and LED LCUs

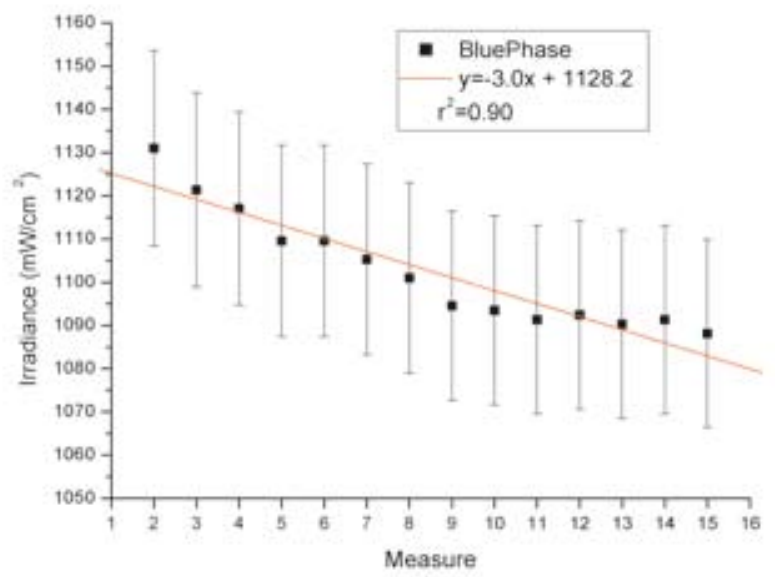

Fig. 5 Total irradiance values of Bluephase with the initial value removed and a new best-fit straight line. The intervals represent instrumental errors.

which have a single LED.

The most probable reason is that there are no circuits to provide a stable electrical intensity. Consequently, tension (and therefore the current) varies and hence the light energy, as the latter is proportional to the tension or current intensity. In the case of LCUs with several LEDs, energy variability is adversely exacerbated as variation in light is multiplied by the number of LEDs. In view of this result, it would be advisable to review the electrical and electronic design of these news LCUs for the correct application of the law of reciprocity between irradiance and irradiation time and for adequate polymerization of dental resin composites.

The lack of energy stability detected in LED LCUs with several LEDs revealed that there was no guarantee that the irradiation time proposed in a protocol would surely and sufficiently provide the total radiant exposure required for the correct polymerization. This result should be taken into account both in works investigating the polymerization kinetics of dental materials as well as when these devices are employed in clinical practice.

\section{ACKNOWLEDGEMENTS}

The authors wish to thank Ivoclar Vivadent (Schaan, Liechtenstein), Mectron (Carasco, Italy), Dentsply (Konstanz, Germany), Satelec (Equipment Division of Acteon Group, Bordeaux, France), Kerr Corporation, (Demetron Division, Danbury, CT, USA), Ultradent Products (South Jordan, UT, USA), and Electro Medical Systems (Nyon, Switzerland) for supplying the LCUs used in this study, and David 
Nesbitt for his assistance with the English version of this manuscript.

\section{REFERENCES}

1) Mills RW. Blue light emitting diodes - another method of light curing. Br Dent J 1995; 178: 169.

2) Mills RW, Jandt KD. Blue LEDs for curing polymer-based dental filling materials. LEOS Newsletter 1998; 12: 9-10.

3) Price RBT, Felix CA, Andreou P. Evaluation of a second-generation LED curing light. J Can Dent Assoc 2003; 69: 666-666i.

4) Jandt KD, Mills RW, Blackwell GB, Ashworth SH. Depth of cure and compressive strength of dental composites cured with blue light emitting diodes (LEDs). Dent Mater 2000; 16: 41-47.

5) Mills RW, Uhl A, Blackwell GB, Jandt KD. High power light emitting diode (LED) arrays versus halogen light polymerization of oral biomaterials: Barcol hardness, compressive strength and radiometric properties. Biomaterials 2002; 23: 29552963.

6) Bennet AW, Watts DC. Performance of two blue light-emitting-diode dental light curing units with distance and irradiation time. Dent. Mater 2004; 20: $72-79$.

7) Uhl A, Sigusch BW, Jandt KD. Second generation LEDs for the polymerization of oral biomaterials. Dent Mater 2004; 20: 80-87.

8) Neumann MG, Miranda WG Jr, Schmitt CC, Rueggeberg FA, Correa IC. Molar extinction coefficients and the photon absorption efficiency of dental photoinitiators and light curing units. J Dent 2005; 33: 525-532.

9) Kirkpatrick SJ. A primer on radiometry. Dent Mater 2005; 21: 21-26.

10) Arikawa H, Kanie T, Fujii K, Ban S. Effect of radiation light characteristics on surface hardness of paint-on resin for shade modification. Dent Mater J
2005; 24: 636-641

11) Uhl A, Mills RW, Rzanny AE, Jandt KD. Time dependence of composite shrinkage using halogen and LED light curing. Dent Mater 2005; 21: 278286.

12) Satsukawa H, Koizumi H, Tanoue N, Nemoto M, Ogino T, Matsumura H. Properties of an indirect composite material polymerized with two different laboratory polymerizing systems. Dent Mater J 2005; 24: 377-381.

13) Rahiotis C, Kakaboura A, Loukidis M, Vougiouklakis G. Curing efficiency of various types of light-curing units. Eur J Oral Sci 2004; 112: 89-94.

14) Halvorson RH, Erickson RL, Davidson CL. Polymerization efficiency of curing lamps: a universal energy conversion relationship predictive of conversion of resin-based composite. Oper Dent 2004; 29: 105-111.

15) Uhl A, Mills RW and Jandt KD. Photoinitiator dependent composite depth of cure and Knoop hardness with halogen and LED light curing units. Biomaterials 2003; 24: 1787-1795.

16) Anonymous. Mind map. Dent Mater 2005; 21: 7-8.

17) Peutzfeldt A, Asmussen E. Resin composite properties and energy density of light cure. J Dent Res 2005; 84: 659-662.

18) Soh MS, Yap AUJ. Influence of curing modes on crosslink density in polymer structures. J Dent 2004; 32: 321-326.

19) Wataha JC, Lewis JB, Lockwood PE, Hsu S, Messer RL, Rueggeberg FA, Bouillaguet S. Blue light differentially modulates cell survival and growth. J Dent Res 2004; 83: 104-108.

20) Shortall AC, Harrington E, Wilson HJ. Light curing unit effectiveness assessed by dental radiometers. J Dent 1995; 23: 227-232.

21) Roberts HW, Vandewalle KS, Berzins DW, Charlton DG. Accuracy of LED and halogen radiometers using different light sources. J Esthet Restor Dent 2006; 18: 214-222. 